\title{
Volitional Control of Autonomic Arousal: A Functional Magnetic Resonance Study
}

\author{
Hugo D. Critchley, ${ }^{*} \dagger$ Raphael N. Melmed,‡ Eric F eatherstone,* Christopher J . Mathias, $†$ and \\ Raymond J . Dolan*'§ \\ *Wellcome Department of Imaging Neuroscience, 12 Queen Square, ION, UCL, London WC1N 3BG, United Kingdom; $¥$ Department of \\ Medicine, Hadassah University Hospital, P.O. Box 12136, J erusalem 91120, Israe; †Autonomic Unit, National Hospital for Neurology \\ and Neurosurgery, and ION, UCL, Queen Square, London, United Kingdom and Department of Neurovascular Medicine Unit, \\ St Mary's Hospital, ICSM, Praed Street, London W2 1NY, United Kingdom; and §Royal Free Hospital,
} UCHSM, UCL, London NW3 2QG, United Kingdom

Received November 28, 2001

Electrodermal activity reflects autonomic sympathetic innervation of dermal sweat glands providing an index of emotion-related bodily states of arousal. Relaxation techniques, which are facilitated by external (bio)feedback of electrodermal activity, can be used by trained subjects to actively control bodily and emotional arousal. Biofeedback relaxation provides an experimental model to explore neural mechanisms contributing to emotional representations and intentional autonomic control. We used functional magnetic resonance imaging (fMRI) to explore neural mechanisms contributing to integration of volitional intent, self-representation, and autonomic states of arousal, embodied within performance of a biofeedback relaxation exercise. Data were obtained from 17 subjects to assess brain activity during relaxation in which a visual index of electrodermal arousal was modulated by accuracy (addition of random "noise") or sensitivity (by scalar adjustments of feedback). A central matrix of cortical, subcortical and brainstem autonomic centres was activated during biofeedback relaxation, as well as regions that mediate visual and somatesthetic representations and executive control. Anterior cingulate, amygdala, and insula activity was modulated by task manipulations that increased demand on processing interoceptive representations, while variation in anterior insula activity reflected an interaction between accuracy and sensitivity of feedback. These findings identify neural substrates that support integration of perceptual processing, interoception, and intentional modulation of bodily states of arousal. $\odot 2002$ Elsevier Science (USA)

Key Words: arousal autonomic; biofeedback; cingulate; brainstem; electrodermal; emotion; feeling states; functional magnetic resonance imaging; skin conductance; thalamus.

\section{INTRODUCTION}

Bodily states of arousal, mediated by the autonomic nervous system, are integral to emotional, cognitive and physical behaviors. Peripheral physiological changes reflect consequences of emotional processing and prospective facilitation of behavior. Thus bodily states of arousal, indexed by autonomic responses, exert feedback influence on subjective emotional experience (Damasio, 1994, 1999), decision-making and memory (Bechara et al., 1997; Cahill, 1997). Given this close interaction between peripheral arousal states with emotion and cognition, a mechanistic understanding of central autonomic control and representation is necessary to fully account for patterns of regional brain activity during emotional and cognitive processing.

Relaxation aimed at decreasing bodily arousal has a recognised therapeutic role in the treatment of emotional disorders as, for example, in exposure therapies for phobic disorders (McGlynn et al., 1999). The ability to control the level of bodily arousal is facilitated by perceptual awareness of covert autonomic responses, as exemplified by enhanced relaxation during biofeedback (e.g., visual or auditory display) of electrodermal activity (EDA) or heart rate (Leahy et al., 1998). Individual differences also influence the success of a relaxation exercise, such as physical build, gender and psychological susceptibility to performance anxiety (Davidson and Schwartz, 1979). Subjective difficulty of a relaxation regimen, closely related to performance anxiety, can be manipulated by degrading accuracy of a feedback signal through the addition of "noise" or by rescaling the feedback signal to emphasise transient skin-conductance responses (SCRs), rather than the basal skin conductance level (SCL). In this context, biofeedback relaxation provides a powerful experimental model to examine brain mechanisms supporting 
central autonomic representation in the context of volitional intent.

Previous neuroimaging studies suggest that brain areas including anterior cingulate and insula provide an interface between cognitive/motivational behaviour and autonomic bodily responses (Critchley et al., 2000a,b, 2001a,b,c; Harper et al., 2000). Activity in these areas, together with striatal and medial temporal lobe structures have modulatory influences on autoregulatory processes within lower-level structures such as hypothalamus and brainstem (Spyer, 1999; Bennarroch, 1997; Harper et al., 2000). Thus, biofeedback relaxation exercises, in which volitional intent, representations of self, and autonomic states of arousal are dynamically integrated, provide a powerful experimental model to examine emotion-related brain mechanisms.

In an earlier positron emission tomography (PET) study of bi ofeedback relaxation, anterior cingulate cortex was implicated as a critical structure in mediating cognitive influences on the level of sympathetic bodily arousal (Critchley et al., 2001c). The present study examines the distributed network of brain areas supporting the volitional regulation of autonomic states, using the higher temporal (and spatial) resolution of fMRI. Subjects were scanned performing variations of a visual-EDA biofeedback relaxation task. We hypothesized that performing an intentional manipulation of sympathetic tone, compared tolow-level baseline intervals, would modulate regional brain activity relating to processing of visual information and representations of volitional intent. By contrast, brain regions involved in the representation of internal bodily states and control of autonomic responses would be modulated both by bi ofeedback task performance and by fluctuations in EDA throughout the experiment. Within the biofeedback relaxation task, crucial experimental variables involved either a manipulation of feedback accuracy or of feedback sensitivity (altering the degree to which the feedback responded to physiological fluctuations in EDA). These manipulations allowed further dissection of component processes underlying bi ofeedback control of autonomic arousal that have wider implications for the understanding of self-regulation of emotional behaviour and neural structures supporting contextual representations of self.

\section{MATERIALS AND METHODS}

\section{Subjects and Functional Task Paradigms}

Seventeen healthy subjects (7F, 10M; mean age \pm SD $33 \pm 3$ years, right-handed) were recruited. Each subject was fully trained before scanning in the performance of a biofeedback relaxation task in which the height of a visually presented "thermometer" represented a logarithmic function of the subjects EDA level.
Subjects were trained to "relax" to decrease the height of the thermometer column to the thermometer bulb. Decreases in column height corresponded to a reduction in EDA, consistent with decrease sympathetic tone. For each subject, at the start of each task, the thermometer height was calibrated to initial electrodermal level and if necessary recalibrated to the maximal EDA level if the subject was not able to decrease sympathetic tone immediately. Thus the thermometer display remained unchanged (taking into account orienting arousal responses to the start of each task) until sympathetic tone decreased. When the subject relaxed sufficiently to decrease the column height to the level of the thermometer bulb, or if $2 \mathrm{~min}$ had elapsed, the display was removed and replaced with a fixation cross. This manner of presentation represented a simple goal-directed performance limited task that did not require working-memory processes. Subjects were also familiarized during training with variations in feedback display encountered during scanning, which enabled them to recognise rapidly the particular task variant when encountered. Debriefing was integral to the training session to facilitate optimal subject performance. Subjects were also interviewed following the scanning session. All subjects gave full, informed, written consent to take part in a study approved by the J oint Research Ethics Committee of the National Hospital for Neurology and Neurosurgery and the Institute of Neurology.

EDA was continuously monitored (SCL 200, Biofeedback Systems, mode 1) from the palmar surface of index and third fingers of left hand. The analogue output from the SCL 200 apparatus, ( $\pm 0.5 \mathrm{~V}$ ) was amplified $\times 30$ before passing though RF filters to the scanner control room and low-pass filtered at $10 \mathrm{~Hz}$. The signal was sampled at $100 \mathrm{~Hz}$. During scanning, each subject performed the bi ofeedback relaxation task every 3 min, signalled by appearance of the thermometer display on the screen. The display remained on the screen until the subject completed the task (forcing the column into the bowl) or until $2 \mathrm{~min}$ had elapsed. During the rest periods of one minute or more between the tasks, the subject was required only to watch a fixation point on the screen, thereby remaining alert in anticipation of the next biofeedback task. This lowlevel "rest" condition provided a contrast in order to examine the main effect of active biofeedback task performance. There were four variations of the task contained within a factorial experimental design that examined the effects of accuracy in feedback (random noise was added to two of the four task conditions) and sensitivity (scaling) of feedback (two of the conditions were rescaled such that a given change in column height represented twice the change in electrodermal ( \pm random fluctuation)) activity in the unscaled task conditions. Each variation was presented twice in a pseudorandom order that was counterbalanced across 
subjects. The control of the biofeedback presentation and continuous, synchronized physiological recording was achieved via a program (developed by EF) written within a Matlab5 platform.

\section{Data Acquisition}

Subjects were scanned during task performance using a Siemens VISION system at 2 Tesla to acquire gradient-echo, echoplanar T2*-weighted images with BOLD (blood oxygenation level dependent) contrast. Each volume comprised $32 \times 3-\mathrm{mm}$ axial scans with 3-mm in-plane resolution and volumes were continuously acquired (trapezoidal sequence) every $3.16 \mathrm{~s}$. Subjects were placed in light head restraint within the scanner to limit head movement during data acquisition. Each run began with 6 "dummy" volumes to allow for T1 equilibration effects, which were subsequently discarded. Additionally a T1-weighted structural image was acquired in each subject for registration with the functional BOLD data. This scan was also used to determine the extent of regional BOLD signal dropout from each subject's functional data set.

I mage Coregistration, Realignment, and Normalization

Data preprocessing to was carried out using SPM99 (Wellcome Department of Cognitive Neurology, London, UK; http:/www.fil.ion.ucl.ac.uk/spm) (Friston et al., (1995a) to correct for head movement and allow functional data sets to be entered into group analyses. All functional volumes, independent of session or paradigm, were realigned to the first volume acquired using rigid-body registration and a mean realigned volume created (Friston et al., 1995b). The subject's T1-weighted structural scan was co-registered to the mean functional volume, and the mean volume used to determine the parameters applied to all volumes during spatial normalisation and re-sampling to a stan- dard template (Friston et al., 1995b, Ashburner and Friston, 1999). As the volume of brain sampled in each study was affected by the position of the subject within the scanner's field of view, we found that the extreme superior and inferior portions of the subject's brain were sparsely sampled. To address this, voxels not sampled in every session were eliminated during normalisation. All functional volumes were then smoothed with an 8mm FWHM Gaussian kernel. Global changes in $\mathrm{fMRI}$ response from scan to scan were removed by proportionally scaling each scan to have a common global mean voxel value.

\section{Statistical Analysis}

Data were analysed using Statistical Parametric Mapping (SPM99) employing the general linear model, where statistical inferences were based on the theory of random Gaussian fields (Friston et al., 1995a). For each subject, a design matrix was constructed to relate the BOLD time course data to the different biofeedback task conditions (modeled as epochs) and including a parametric regressor of the continuous EDA level over the whole of the experiment. Lag in BOLD signal changes relative to task variable was approximated by convolution of box-car epochs for the tasks, and the EDA regressor, with a canonical haemodynamic response function. This effectively implemented a leastsquares deconvolution of enduring components of the haemodynamic response to individual events and epochs to all ow examination of regional changes in neural activity underlying these haemodynamic responses. Using multiple regression analyses, statistical parametric maps (SPMs) were constructed representing the association between the observed time series and one or a linear combination of the regressors for each subject. Thus, we derived SPMs for activity relating to; (1) main effect of performance of biofeedback relaxation tasks; (2) changes in EDA over the whole experiment;

FIG. 1. Diagram of analytic design. During $f M R I$, subjects performed two repetitions of four variations of a biofeedback relaxation exercise, interspersed with rest periods. Electrodermal activity, EDA, was monitored throughout the scanning period and a logarithmic function of EDA represented the visual feedback given to the subject in the form of a "thermometer" which decreased in column height with decreases in EDA. This relationship between feedback and EDA was either accurate (Tasks A and C) or degraded by the addition of a random fluctuation in column height, adding "noise" to the feedback of physiological arousal level (Tasks B and D). Two different levels of scaling of feedback were also included in the factorial design. Tasks $C$ and $D$ scaled to half that of tasks $A$ and $B$, such that for an equivalent decrease in column height the subject needed to decrease their electrodermal arousal (log EDA) by a factor of 2 . Analyses tested for regional brain activity (1) associated with performance of bi ofeedback relaxation (tasks vs rest); (2) covarying EDA throughout the experiment and, within the factorial structure of the tasks for; (3) main effect of accuracy/noise of feedback (intended to highlight activity reflecting greater reliance on intero- versus exteroceptive representations of bodily arousal; (4) main effect of scaling of feedback (intended to highlight activity reflecting representation of perceptual characteristics of feedback) and; (5) interaction between accuracy and scaling of feedback, intended to highlight activity directly underlying volitional and perceptual influences on the control of bodily arousal.

FIG. 2. Task performance data in one subject. The figure plots the BOLD signal changes for three brain regions (right anterior cingulate, insula and thalamus), the continuous recording of EDA and the height of the biofeedback display (red thermometer) for one subject over the course of the experiment. The x-axis represents timings of scan volume acquisitions (TR 3.16 s) relative to the tasks and rest periods. Numbers within the lower plot indicate the task performed by the subject (see Fig. 1 and text). The feedback signal was scaled at the start of the task to the subject's concurrent EDA level and began to fall when EDA decreased. Fluctuations in feedback signal and scaling of the feedback can be viewed in relation to the slope of the lower plots relative to the continuous EDA recording. 

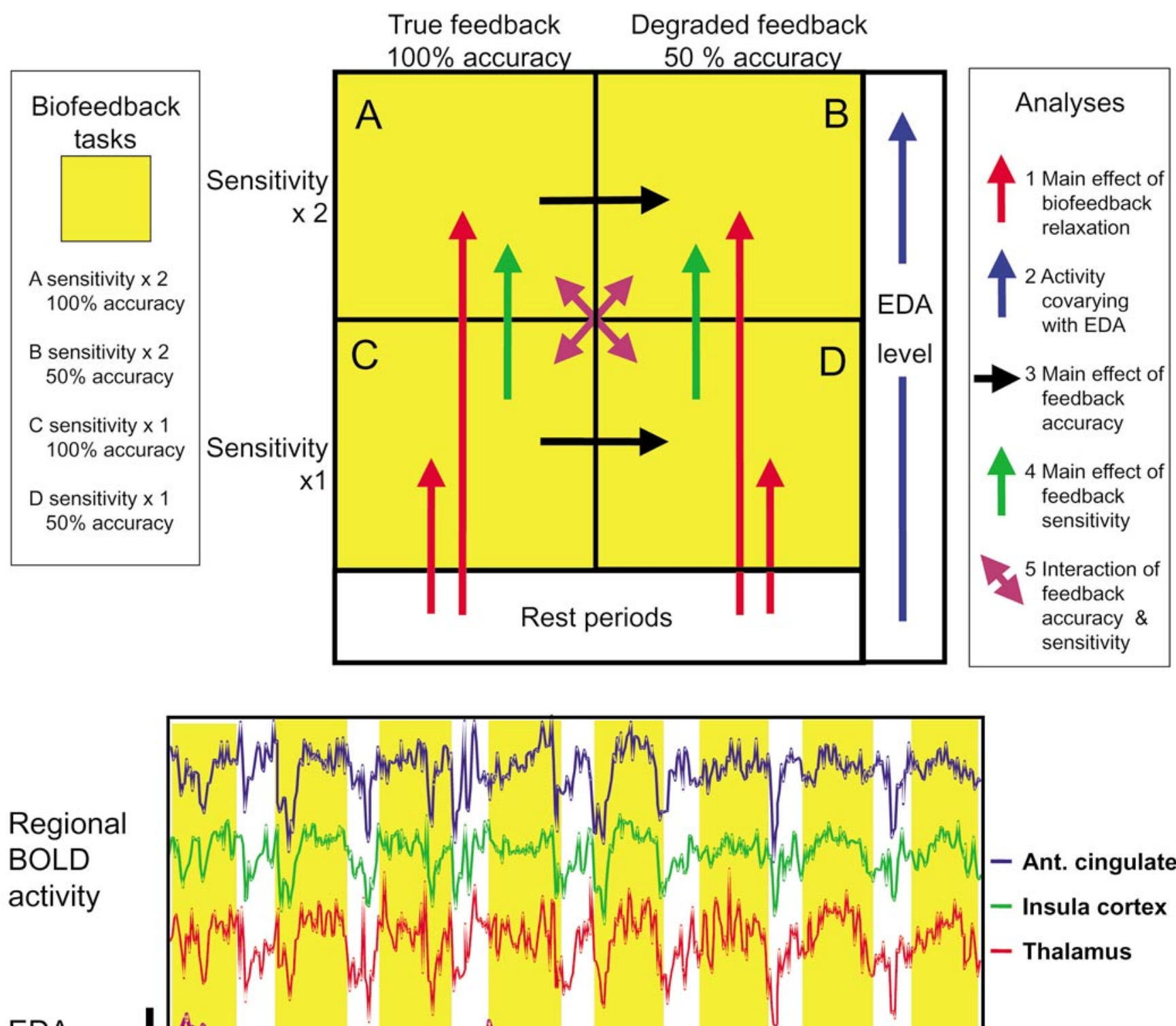

Regional BOLD activity signal

Biofeedback 2
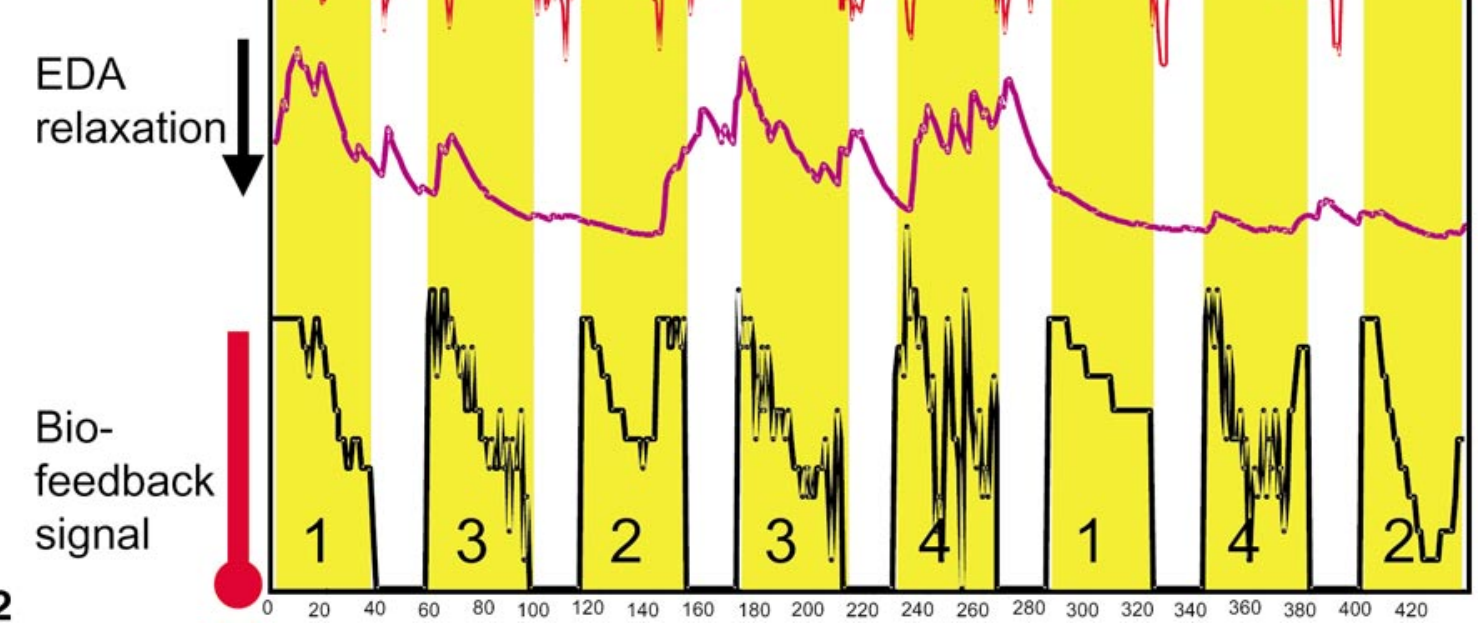

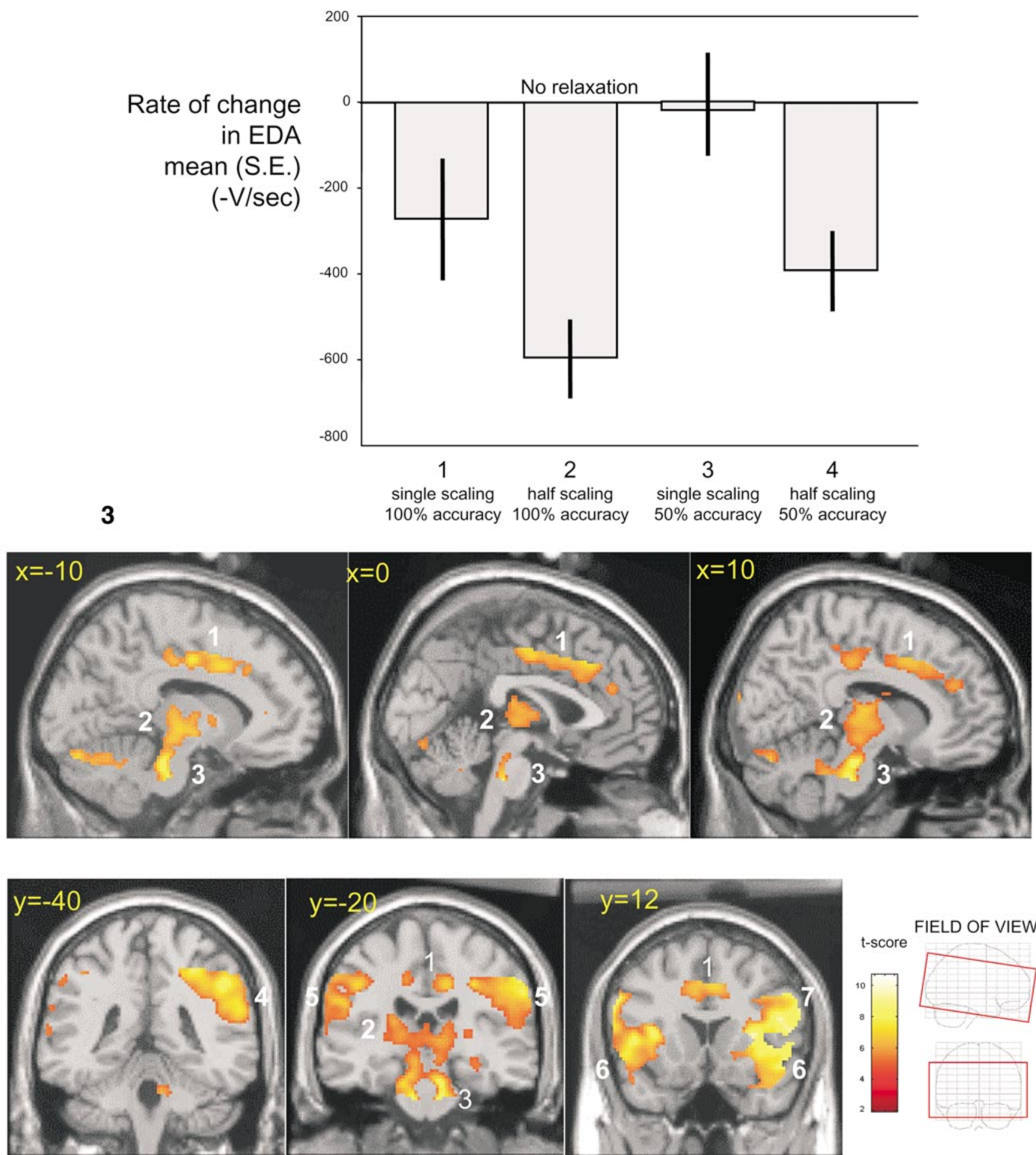

FIELD OF VIEW t-score

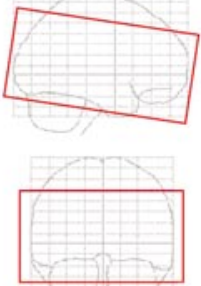

FIG. 3. Group data for rates of relaxation during bi ofeedback task performance. The rate at which subjects relaxed, i.e., decreased EDA arousal during biofeedback tasks, was derived from the change in EDA level over a 1-min period, $30 \mathrm{~s}$ into each task. The different tasks were associated with different rates of relaxation, with significantly faster rates of relaxation achieved when more feedback was rendered less sensitive by resealing the feedback to half. The addition of random noise into the feedback signal was associated with a strong trend in impairment of relaxation, and resulted in no significant relaxation when subjects performed biofeedback relaxation at the more sensitive task-scaling.

FIG. 4. Regional brain activity associated with performance of biofeedback relaxation. The figure illustrates group data, plotted on a normalized template brain, derived from random effects analysis and associated with performance of bi ofeedback relaxation tasks, relative to rest periods. Data are presented on a normalized template brain scan, color-scaled according to the significance of the effect and thresholded for presentation purposes at $\mathrm{P}<0.001$, uncorrected. Parasagittal sections (labelled according to distance from midline) and coronal sections (labelled in $\mathrm{mm}$ from anterior commissure) have been chosen to illustrate (1) cingulate, (2) thalamic and hypothalamic activity, (3) pontine activity, (4) right parietal, (5) bilateral somatosensory, (6) anterior insula, and (7) dorsolateral prefrontal cortical activity. The color bar reflects the t scores of bi feedback task-related activity. The brain coverage of the 32 slice data acquisition is illustrated by a red box denoting field of view on sagittal and coronal "glass brain" projections. 
(3) main effect of degraded feedback signal; (4) main effect of changing sensitivity of feedback and; (5) interaction between feedback accuracy and sensitivity. These SPMs, derived from individual subject data, were then entered into a random-effects second-level analysis wherein significance of regional contrast-related activity at the population level was tested with a $\mathrm{T}$ statistic to give a SPM $\{\mathrm{T}\}$. Activity at this second level reaching statistical significance at $P<0.05$, corrected for whole brain or small volume of predicted regions of interest (Worsley et al., 1996). The coordinates of a priori regions of interest for the main effect of task performance were derived from earlier independent observations of regional activity associated with modulation of autonomic responses and visceral representation. These were anterior cingulate (Critchley et al., 2000a, 2001a,b,c; Harper et al., 2000), amygdala and basal ganglia (Critchley et al., 2001c), thalamus and hypothalamus (Harper et al., 2000). Regional corrections for small volumes were subsequently based on activity observed as a main effect of task performance, for a minimum volume of $100 \mathrm{~mm}^{3}$. Descriptions of anatomical location were determined using average structural MRIs normalized to standard space (Talairach and Tournoux, 1988), using the atlas of Duvernoy (1991).

\section{RESULTS}

Subjects were interviewed after scanning to determine the strategies they employed for biofeedback relaxation and their subjective impressions of the individual tasks. All subjects recognized the presence of noise and sensitivity change in bi ofeedback, consistent with their training experience. During scanning, subjects reported using the same strategies for relaxation as they had used during training, which consisted of reducing skeletomotor tone/movement and intentionally "feeling calm" (other insights included "feeling heavy" or "floppy", "going blank", "letting it happen"). E pisodic recall of experience with individual tasks was poor following the scanning session, but evidence from training sessions suggested that subjective performance difficulty was closely related to task performance. The ( $1 \mathrm{~min}$ ) inactive periods between biofeedback relaxation tasks were viewed as "breaks." Most subjects also reported increasing anticipation of the next experimental task during this period. Onset of scanning produced increased subjective alertness/ arousal and a delay in achieving a decrease in thermometer height due to corresponding EDA activity increase. Order effects of this type were accounted for by the counterbalanced study design.

Performance of biofeedback relaxation was determined as the rate at which subjects were able to decrease EDA over the course of the experiment. Relaxation rates were derived from a period of $1 \mathrm{~min}$ beginning 30 s into the task, excluding initial arousal effects engendered by task engagement. Subjects relaxed at a significantly slower rate when feedback was scaled to be more sensitive (i.e., when the thermometer height reflected half the decrement in $\log (E D A)$ ) $(T(62)=3.07, P<0.005)$. Similarly, there was a strong trend for addition of random "noise" to the feedback signal to impair relaxation rate $(T(62)=1.96$; $\mathrm{P}=$ 0.054). The combination of decreased accuracy and increased sensitivity was associated with an absence of significant relaxation. We observed no significant interaction between accuracy and sensitivity of feedback in the rates at which subjects reduced EDA arousal $(F(1)=0.08$, n.s.) (Fig. 3).

To identify the neuroanatomical matrix supporting biofeedback relaxation, we first tested for activity re lating to performance of biofeedback tasks (all) compared to rest periods between the tasks. We observed widespread activity, consistent with activation of a distributed neural system, involving regions implicated in visual movement processing (extrastriate cortex (V5)), attention and response selection (dorsolateral prefrontal cortex, anterior cingulate cortex, inferior parietal lobule, thalamus), somatic/visceral representations of bodily state (bilateral insula, primary and secondary somatosensory cortices, thalamus), representations of motivational goals (basal ganglia, medial temporal lobe, thalamus) as well as areas implicated in autoregulatory control of sympathetic autonomic responses (substantia nigra, hypothalamic nuclei and pons) (Fig. 4, Table 1). We observed no significant decreases in activity associated with performing the biofeedback relaxation tasks.

We next tested for activity relating to increases and decreases in sympathetic tone across the whole experiment. In a previous study we described anatomical overlap in brain activity associated with generation and representation of EDA (Critchley et al., 2000b). Therefore, we tested for regional activity associated with this feedback "loop" that was synchronous with the EDA (the EDA regressor was convolved with a canonical haemodynamic response function). Activity in anterior cingulate, dorsolateral prefrontal cortex, basal ganglia, somatosensory cortex, thalamus and pons covaried negatively with level of electrodermal arousal. (Fig. 5; Table 1). In other words, these areas became more active when level of sympathetic arousal decreased over the course of the experiment. Activity associated with increasing sympathetic arousal (EDA) did not reach significance.

Within the factorial experimental design, we tested for effects on regional brain activity of modulations in noise and sensitivity of the biofeedback signal. We reasoned that the noise manipulation, altering feedback veracity, may highlight processing of discrepancies between "corrupted" exteroceptive and veridical interoceptive representations of relaxation-state. By 
TABLE 1

Regional Brain Activity Associated with Performance of Biofeedback Tasks

\begin{tabular}{|c|c|c|c|}
\hline Region & Side & Coordinates of peak activity & T score \\
\hline Extrastriate (v5) & $\mathrm{R}$ & $50,-68,-2$ & 10.93* \\
\hline Inferior occipital/fusiform gyrus & $\mathrm{L}$ & $-46,-74,-12$ & $8.11 *$ \\
\hline Fusiform gyrus & $\mathrm{R}$ & $42,-62,-16$ & $8.01 *$ \\
\hline Middle frontal gyrus & $\mathrm{R}$ & $56,10,34$ & 10.68* \\
\hline Inferior frontal gyrus & L & $-60,6,22$ & $10.00 *$ \\
\hline Inferior frontal gyrus & $\mathrm{R}$ & $50,16,18$ & $8.91 *$ \\
\hline Precentral gyrus & L & $-52,-4,40$ & $9.17 *$ \\
\hline Precentral gyrus & $\mathrm{R}$ & $44,-10,40$ & 7.10* \\
\hline Postcentral gyrus somatosensory cortex & $\mathrm{R}$ & $54,-16,22$ & 8.36* \\
\hline Inferior parietal lobule & $\mathrm{R}$ & $50,-40,40$ & 7.83* \\
\hline Insula & $\mathrm{R}$ & $32,16,8$ & $9.08 *$ \\
\hline Insula & $\mathrm{R}$ & $48,8,6$ & $8.34 *$ \\
\hline Anterior cingulate & - & $0,24,40$ & $5.97 \dagger$ \\
\hline Mid cingulate & - & $2,-14,48$ & $5.91 \dagger$ \\
\hline Amygdala & $\mathrm{R}$ & $26,-4,-26$ & $4.79+$ \\
\hline Putamen/claustrum & $\mathrm{R}$ & $28,0,8$ & $5.88 \dagger$ \\
\hline Putamen & L & $-28,-8,4$ & $5.01 \dagger$ \\
\hline Thalamus (mediodorsal nucleus) & $\mathrm{R}$ & $6,-14,6$ & $5.63 \dagger$ \\
\hline Thalamus (ventrolateral) & $\mathrm{L}$ & $-16,-10,-2$ & $5.46 \dagger$ \\
\hline Thalamus (ventrolateral) & $\mathrm{R}$ & $14,-12,0$ & $5.17 \dagger$ \\
\hline Hypothalamus & L & $-6,-6,-6$ & $5.13 \dagger$ \\
\hline Sustantia nigra/red nucleus & L & $-8,-18,-2$ & $5.17 \dagger$ \\
\hline Substantia nigra & $\mathrm{R}$ & $10,-20,-8$ & $4.96 \dagger$ \\
\hline Dorsal pons & $\mathrm{R}$ & $10,-22,-24$ & $7.59 *$ \\
\hline Dorsal pons & L & $-6,-28,-26$ & 7.10* \\
\hline \multicolumn{4}{|l|}{ Brain region covarying with electrodermal activity } \\
\hline Globus pallidus/putamen & L & $-18,-4,2$ & $5.34 \dagger$ \\
\hline Post central gyrus somatosensory cortex & $\mathrm{R}$ & $52,-24,42$ & $4.79 \dagger$ \\
\hline Secondary somatosensory cortex & & $-54,-12,6$ & $4.22 \dagger$ \\
\hline Anterior cingulate & - & $6,18,22$ & $4.66 \dagger$ \\
\hline Anterior cingulate & $\mathrm{R}$ & $6,30,28$ & $4.56 \dagger$ \\
\hline Mid cingulate & L & $-6,-2,46$ & $4.24 \dagger$ \\
\hline Medial prefrontal cortex/anterior cingulate & L & $-10,46,20$ & $4.22 \dagger$ \\
\hline Thalamus (mediodorsal nucleus) & $\mathrm{R}$ & $6,-10,16$ & $3.99+$ \\
\hline Lateral pons & $\mathrm{R}$ & $8,-18,-18$ & $3.88 \dagger$ \\
\hline \multicolumn{4}{|l|}{ Main effect of noisy, degraded feedback } \\
\hline Amygdala & $\mathrm{R}$ & $26,-4,-12$ & $6.28 \dagger$ \\
\hline Insula & $\mathrm{R}$ & $34,-2,16$ & $6.26 \dagger$ \\
\hline Insula & L & $-42,4,8$ & $4.74 \dagger$ \\
\hline Hypothalamus/substantia nigra & - & $2,-12,-10$ & 4.93 \\
\hline Extrastriate cortex v5 & $\mathrm{R}$ & $38,-64,2$ & $5.11 \dagger$ \\
\hline Extrastriate cortex v5 & $\mathrm{R}$ & $50,-68,16$ & $4.88 \dagger$ \\
\hline Postcentral gyrus somatosensory cortex & $\mathrm{R}$ & $66,-16,34$ & $4.83 \dagger$ \\
\hline Anterior cingulate & - & $2,18,30$ & $4.52 \dagger$ \\
\hline Anterior cingulate & - & $2,-12,-8$ & $4.49 \dagger$ \\
\hline Anterior cingulate & - & $0,12,38$ & $4.33 \dagger$ \\
\hline Anterior cingulate & - & $6,28,34$ & $3.89 \dagger$ \\
\hline \multicolumn{4}{|c|}{ Interaction between noise and sensitivity of bi ofeedback } \\
\hline Lingual gyrus & L & $-28,-46,-4$ & $4.88 \dagger$ \\
\hline Anterior Insula & L & $-44,20,0$ & $4.50 \dagger$ \\
\hline
\end{tabular}

Note. Activity significant to $\mathrm{P}<0.001$, uncorrected; *Significant to $\mathrm{P}<0.05$, whole brain correction for multiple comparisons; $\uparrow$ significant to $\mathrm{P}<0.05$, small volume for region of interest.

contrast, the sensitivity manipulation was aimed at providing accurate feedback with altered perceptual characteristics. However, it is acknowledged that this manipulation also increased the susceptibility of the bi ofeedback system to "self-generated noise" from transient SCRs. We also tested for an interaction between these two manipulations. The addition of random noise was associated with increased activity in right amygdala, insula, anterior cingulate, a hypothalamic region and extrastriate visual cortex (v5), (Fig. 6, Table 1). There were no significant decreases in activity. Altering the sensitivity of feedback was not associated with a significant main effect. Since both addition of noise and increased feedback sensitivity impaired rates at 


\section{$4,24,27$ Cingulate $t=4.66$}

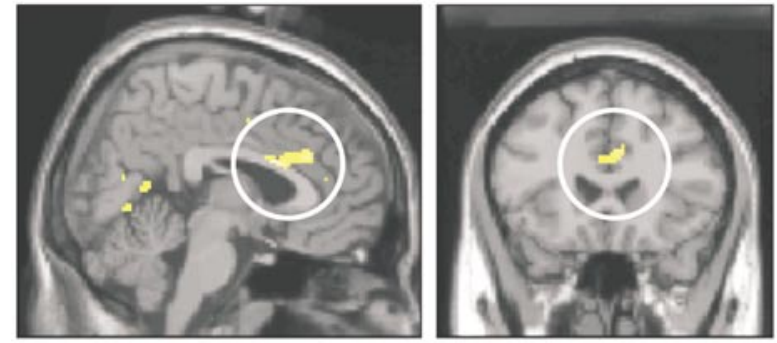

\section{$-18,5,2$ Basal ganglia $t=5.34$}
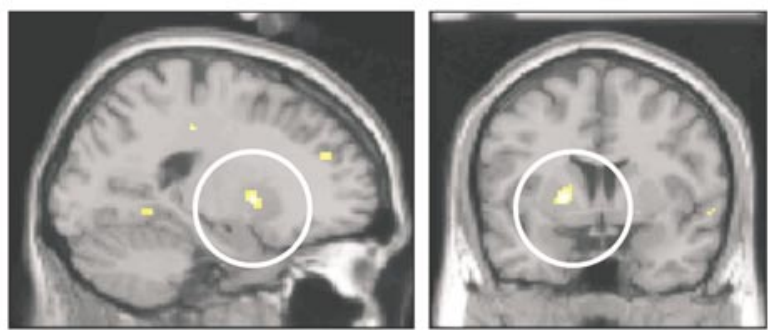

$52,-24,42$ Somatomotor $t=4.79$
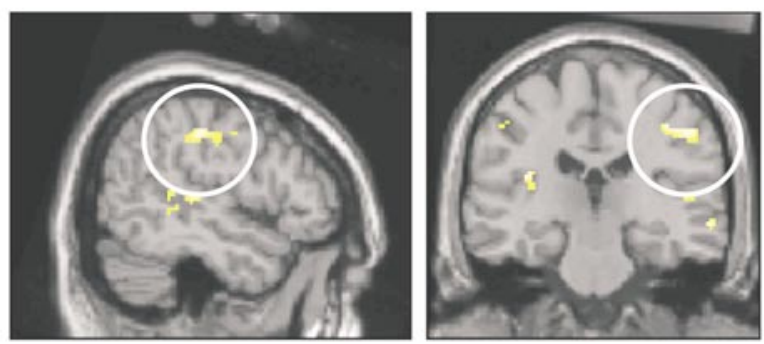

FIG. 5. Brain regions covarying with decreases in sympathetic (EDA) arousal. Group data is plotted on coronal, axial, and parasagittal sections of a template brain, illustrating changes in regional activity within somatosensory cortex, anterior cingulate cortex and putamen, covarying negatively with electrodermal arousal over the course of the experiment (random effects analysis, $\mathrm{P}<0.001$, uncorrected for illustrative purposes).

which subjects decreased EDA arousal during biofeedback relaxation, particularly when these conditions were combined, we tested for activity reflecting the interaction between feedback accuracy and sensitivity. We observed significant activity reflecting this interaction (i.e., [more versus less sensitive tasks with noise] versus [more versus less sensitive tasks without noise]) in left anterior insula (Table 1).

\section{DISCUSSION}

Our study addresses the functional neuroanatomy supporting integration of cognitive and perceptual processing with intentional set in the control of peripheral sympathetic arousal. We identify a matrix of cortical and subcortical brain regions associated with biofeedback relaxation and note that these regions are also implicated in processing of visual information (v5 cortex), representation of bodily states (e.g., thalamus, insula and somatosensory cortices) and autoregulatory control of peripheral autonomic responses (hypothalamic area and pons). Biofeedback relaxation was also associated with increased activity in brain regions such as dorsolateral prefrontal, anterior cingulate and parietal cortices, amygdala and basal ganglia that may support integration of volitional processing, expressed in the intention to relax, with indices of bodily responses. Specifically these regions may mediate interactions between external (biofeedback information)

\section{$2,18,28$ Cingulate $t=4.81$}
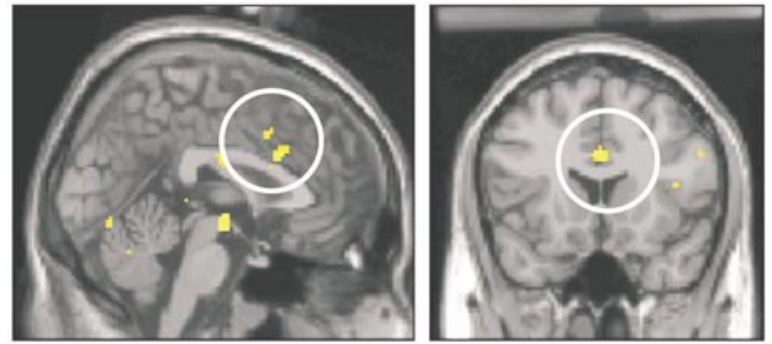

\section{$26,-4,12$ Amygdala $t=6.28$}
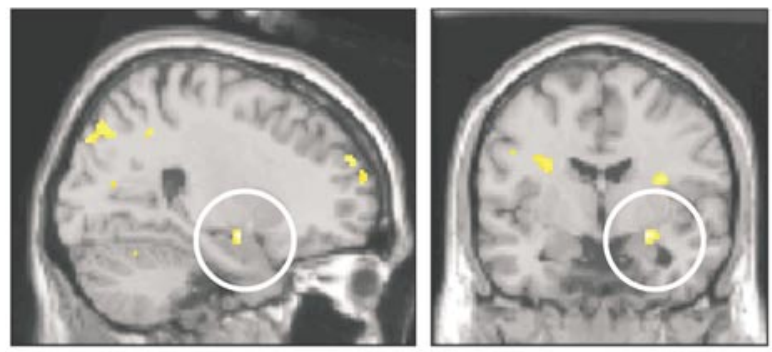

\section{$0,-12,-8$ Hypothalamus $t=4.82$}
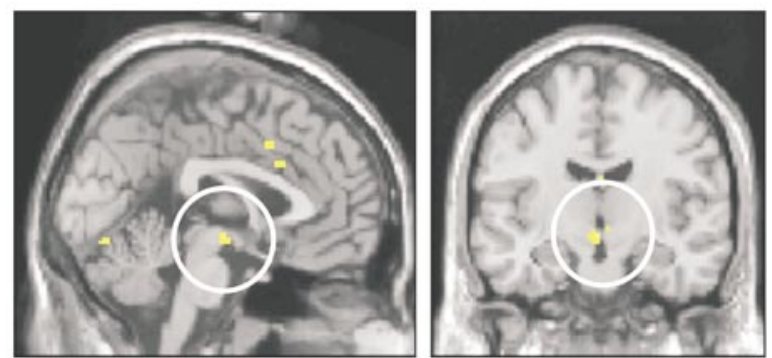

FIG. 6. Brain regions reflecting the main effect of noise in the bi ofeedback signal. The main effect of decreased accuracy of bi ofeedback, achieved by adding random fluctuation to the feedback signal, was associated with increased activity in anterior cingulate, right amygdala, and hypothalamus. The figure illustrates the localization of significant group activity in these brain regions on coronal, axial and parasagittal sections of a template brain. 
and internal sensory feedback (interoceptive sensory information) with attentional and motivational states. Evidence for this latter proposal includes data implicating dorsolateral prefrontal cortex in response-selection, on-line information processing and self-monitoring (Rowe et al., 2000; Petrides et al., 1993; Henson et al., 2000), while anterior cingulate is implicated in error-detection, response inhibition, attentional selection, representation of subjective experience and, more recently, in integrating bodily responses with behavioural demands (Carter et al., 1998; Rubia et al., 2001; Pardo et al., 1990; Lane et al., 1997; Critchley et al., 2000a, 2001a,b,c). The distributed matrix we describe provides a likely substrate for contextual control and feedback representation of states of bodily arousal; processes that are critical to theoretical models of emotion (Damasio, 1994, 1999).

A discrete subgroup of these regions reflected modulation of EDA-indexed arousal across all tasks, implying an obligatory role in representation of electrodermal arousal. These regions include anterior cingulate, right somatosensory cortex and basal ganglia (putamen).

Previous studies have observed increases in anterior cingulate activity with cognitively-driven changes in bodily states of arousal (Critchley et al., 2000a, $2001 a, b, c)$. Anterior cingulate cortex is also implicated in control of cardiovascular states of arousal during effortful behaviour (Critchley et al., 2000a, 2001b). Activity in this region is modulated by both level of electrodermal arousal and risk during reward anticipation (Critchley et al., 2001a). In an earlier PET study of biofeedback relaxation, enhanced anterior cingulate activity was associated with intention to relax and the interaction between relaxation and presence of feedback. In the present experiment involving intentional relaxation, the observation of enhanced anterior cingulate activity associated with both decreasing electrodermal arousal and manipulation of feedback, suggests a tuning of cingulate arousal-related responses to contextual demands (Critchley et al ., 2001c). Basal ganglia activity was also observed in this earlier study of bi ofeedback relaxation, where activity in globus pallidus was associated with intention to relax and interpreted as reflecting decreases in skeletomotor activity during the relaxation process (Critchley et al., 2001c). The present study extends this observation by observing selective putaminal activity in association with the representation of bodily arousal. These findings indicate that activity associated with decreases in EDA across the experiment reflects modulatory influences of bodily arousal on brain regions mediating intentional and goal-directed aspects of behavior.

The manipulations of accuracy and sensitivity of feedback were directed at highlighting distinct influences on the control of bodily states of arousal. The hypothesized consequence of the addition of "noise" (random fluctuation) to the feedback signal is that subjects place greater reliance on internal bodily cues, relative to external feedback, in order to achieve successful relaxation. The reduced rate of relaxation across subjects observed during noisy conditions suggests that mismatch of externally driven and interoceptive representations of bodily arousal impairs cognitively driven modulation of EDA. The observation of parallel increases in activity in insula, right amygdala, anterior cingulate, and hypothalamus provides are consistent with increased demand on contextual representation of internal bodily states. An alternative, but nevertheless complimentary, explanation is that activity in these regions reflects "stress" in response to the degraded quality of feedback, a proposal consistent with putative roles for the same regions in signalling aversion or deviations in congruency (Buchel et al., 1998; MacDonald et al., 2000). However, activity in these areas was not associated with increased task demands per se, as there was no main effect of changing the sensitivity of the feedback signal. Consequently, a third account would suggest that enhanced activity in insula, amygdala and cingulate activity during "noisy" tasks reflects conflict-processing between representations of internal state and external indices of arousal. This is also supported by our finding that insular cortex activity was associated with an interaction between accuracy and sensitivity of the biofeedback task. More specifically, left insula activity was greater in the comparison of more versus less sensitive feedback when the feedback was degraded than when feedback was accurate. This observation indicates a role of insula in perceptual integration of external sensory information with representation of internal bodily states, a "cognitive" elaboration of its putative viscerosensory function suggested by earlier observations (Critchley et al., 2001a).

Brain areas implicated in autoregulation of bodily arousal (Critchley et al., 2001b) were not, in general, influenced by manipulations in accuracy and sensitivity of the biofeedback signal. Thus, regions such as pons were highlighted as components of the biofeedback matrix, whose activity correlated with changes in EDA arousal but was not influenced by changes in feedback. However, activity in a hypothalamic/substantia nigra area was influenced by noise in the biofeedback signal suggesting, that this area may be the lowest level through which autonomic regulation of bodily states of arousal may be directly modulated by descending cognitive influences. In contrast, cortical areas implicated in the representation of bodily states, such as somatosensory cortices and insula (Critchley et al., 2001b) were modulated by diminished accuracy in the biofeedback signal, suggesting that interoceptive representations in these brain regions were modulated by cognitive processing of external information, similar perhaps to mechanisms mediating cognitive influences 
on pain thresholds (Petrovic et al., 2000). Areas such as dorsolateral prefrontal and right parietal cortices, which were activated by task performance, but not influenced by level of autonomic arousal or manipulation in biofeedback, are likely to reflect an attentional engagement during bi ofeedback. While previous studies imply a more direct link between autonomic arousal and activity in prefrontal and right parietal cortices, the present study highlights our earlier proposal that "intention to relax" is mediated by activity changes within these regions (Critchley et al., 2001a,c). However, our findings especially highlight the role of areas such as cingulate and insula in mediating the interface between cognitive intent and changes in bodily arousal, as well as supporting the interaction between perceptual, cognitive and interoceptive representations that in turn influence autonomic responses.

In conclusion, our findings detail activity associated with cognitively driven sympathetic relaxation facilitated by bi ofeedback of EDA, and implicate a matrix of cortical and subcortical brain regions in mediating the integration of bodily states of arousal with cognitive and perceptual processing. Of crucial importance is the observation that activity within anterior cingulate, insula and somatosensory cortices reflects level of sympathetic arousal. Anterior cingulate, cortex also increases its activity during presentation of degraded feedback but not manipulation of task-demand per se. Thus, if the anterior cingulate is involved, as earlier studies indicate (Critchley et al., 2000a,2001a,b,c), in the contextual generation of bodily states of autonomic arousal, its role is more pronounced when external information conflicts with internal states. The identification of brain mechanisms involved in generation, modulation and feedback representation of bodily states of arousal has wider implications in so far as they also crucial aspects of emotional experience or so-called "feeling-states," which provide important guides to cognitive and motivational behaviours (Damasio, 1999; Bechara et al., 1997; Cahill et al., 1997).

\section{ACKNOWLEDG MENTS}

This work was supported by a Programme Grant to R.J .D. from the Wellcome Trust. H.D.C. is supported by a Project Grant from the Brain Research Trust to H.D.C., R.J .D., and C.J .M. R.N.M. was supported by a a Wellcome Short-Term Travel Fellowship and an award from the Paul Balint Trust during his sabbatical to the Institute of Neurology. Correspondence and requests for materials should be addressed to Hugo Critchley, Wellcome Department of Cognitive Neurology, ION, UCL, Queen Square, London WCIN 3BG, United Kingdom h.critchley@il.ion.ud.ac.uk.

\section{REFERENCES}

Ashburner, J ., and Friston, K. 1999. Nonlinear spatial normalization using basis functions. Hum. Brain Mapp. 7: 254-260.
Bechara, A., Damasio, H., Tranel, D., and Damasio, A. R. 1997. Deciding advantageously before knowing the advantageous strategy. Science 275: 1293-1295.

Bennarroch, E. E. 1997. Functional anatomy of the central autonomic network. In Central Autonomic Network: Functional Organization and Clinical Correlations (E. E. Bennarroch, Ed.), pp. 29-60. Futura, Armonk, NY.

Buchel, C., Morris, J., Dolan, R. J ., and Friston, K. J . 1998. Brain systems mediating aversive conditioning: An event-related fMRI study. Neuron 20: 947-957.

Cahill, L. 1997. The neurobiology of emotionally influenced memory Implications for understanding traumatic memory. Ann. N.Y. Acad. Sci. 821: 238-246.

Carter, C. S., Braver, T. S., Barch, D. M., Botvinick, M. M., Noll, D., and Cohen, J. D. 1998. Anterior cingulate cortex, error detection, and the online monitoring of performance. Science 280: 747-749.

Critchley, H. D., Corfield, D. R., Chandler, M. P., Mathias, C. J ., and Dolan, R. J . 2000a. Cerebral correlates of autonomic cardiovascular arousal: A functional neuroimaging investigation. J . Physiol. 523: $259-270$

Critchley, H. D., Mathias, C. J., and Dolan, R. J . 2001a. Neural activity relating to reward anticipation in the human brain. Neuron 29: 537-545.

Critchley, H. D., Elliott, R., Mathias, C. J ., and Dolan, R. J . 2000b. Neural activity relating to the generation and representation of galvanic skin conductance response: A functional magnetic imaging study. J . Neurosci. 20: 3033-3040.

Critchley, H. D., Mathias, C. J ., and Dolan, R. J . (2001b) Neural correlates of first and second-order representation of bodily states. Nature Neurosci. 4: 207-212.

Critchley, H. D., Melmed, R. N., Featherstone, E., Mathias, C.J ., and Dolan, R. J . 2001c. Brain activity during biofeedback relaxation: a functional neuroimaging investigation. Brain 124: 1003-1012.

Damasio, A. R. 1994. Descartes' Error: Emotion, Reason and the Human Brain. Grosset/Putnam, New York.

Damasio, A. R. 1999. The Feeling of What Happens: Body and Emotion in the Making of Consciousness. Harcourt Brace, New York.

Davidson, R. J ., and Schwartz, G. E. 1979. Patterns of cerebral lateralization during cardiac biofeedback versus the self-regulation of emotion: sex differences. Psychophysiology 13: 62- 68

Duvernoy, H. M. 1991. The Human Brain: Surface ThreeDimensional Sectional Anatomy and MRI. Springer-Verlag, New York.

Friston, K., Ashburner, J ., Frith, C. D., Poline, J .-B., Heather, J . D., and Frackowiak, R. S. J . 1995. Spatial registration and normalization of images. Hum. Brain Mapp. 2: 165-189.

Friston, K., Holmes, A. P., Worsley, K. J ., Poline, J .-P., Frith, C. D., and Frackowiak, R. S. J. 1995. Statistical parametric maps in functional imaging: A general linear approach. Hum. Brain Mapp. 2: 189-210.

Friston, K. J ., Holmes, A. P., Price, C. J ., Buchel, C., and Worsley, K. J. 1999. Multisubject fMRI studies and conjunction analyses. Neuroimage 10: 385-396.

Harper, R. M., Bandler, R., Spriggs, D., and Alger, J. R. 2000. Lateralized and widespread brain activity during transient blood pressure elevation revealed by magnetic resonance imaging J . Comp. Neurol. 417: 195-204.

Henson, R. N., Rugg, M. D., Shallice, T., and Dolan, R. J . 2000. Confidence in recognition memory for words: Dissociating right prefrontal roles in episodic retrieval J . Cogn. Neurosci. 12: 913923.

Lane, R. D., Fink, G. R., Chau, P. M., and Dolan, R. J . 1997. Neural activation during selective attention to subjective emotional re sponses. Neuroreport 8: 3969-3972. 
Leahy, A., Clayman, C., Mason, I., Lloyd, G., and Epstein, O. 1998. Computerised biofeedback games: A new method for teaching stress management and its use in the irritable bowel syndrome. J . R. Coll. Physicians (London) 32: 556-559.

MacDonald, A. W., 3rd, Cohen, J. D., Stenger, V. A., and Carter, C. S. 2000. Dissociating the role of the dorsolateral prefrontal and anterior cingulate cortex in cognitive control. Science 288: 1835-1888.

McGlynn, F. D., Moore, P. M., Lawyer, S., and Karg, R. 1999. Relaxation training inhibits fear and arousal during in vivo exposure to phobia-cue stimuli. J . Behav. Ther. Exp. Psychiatry 30: 55-56.

Pardo, J . V., Pardo, P. J ., J aner, K. W., and Raichle, M. E. 1990. The anterior cingulate cortex mediates processing selection in the Stroop attentional conflict paradigm. Proc. Natl. Acad. Sci. USA 87: 256-259.

Petrides, M., Alivisatos, E., Meyer, E., and Evans, A. C. 1993 Functional activation of the human frontal cortex during performance of verbal working memory tasks. Proc. Natl. Acad. Sci. USA 90: 878- 882.

Petrovic, P., Petersson, K. M., Ghatan, P. H., Stone-Elander, S., and Ingvar, M. 2000. Pain-related cerebral activation is altered by a distracting cognitive task. Pain 85: 19-30.
Rowe, J. B., Toni, I., J osephs, O., Frackowiak, R. S. J ., and Passingham, R. E. 2000. The prefrontal cortex: Response selection or maintenance within working memory? Science 288: 1656-1664.

Rubia, K., Russell, T., Overmeyer, S., Brammer, M. J ., Bullmore, E. T., Sharma, T., Simmons, A., Williams, S. C., Giampietro, V., Andrew, C. M., and Taylor, E. 2001. Mapping motor inhibition: Conjunctive brain activations across different versions of go/no-go and stop tasks. Neuroimage 13: 250-261.

Spyer, K. M. 1999. Central nervous control of the cardiovascular system. In Autonomic Failure: A Textbook of Clinical Disorders of the Autonomic Nervous System (C. J. Mathias and R. Bannister, Eds.), pp. 45-55. Oxford Univ. Press, Oxford.

Talairach, J ., and Tournoux, P. 1988. Co-planar Stereotaxic Atlas of the Human Brain. Theime, Stuttgart.

Worsley. K. J ., Marrett, P., Neelin, A. C., Friston, K. J ., and Evans, A. C. 1996. A unified statistical approach for determining significant signals in images of cerebral activation. Hum. Brain Mapp. 4: $58-73$. 